\title{
ANÁLISIS DEL MARCO DE ACTUACIÓN DE SOCIEDADES OFF SHORE EN ARGENTINA: ESPECIAL MIRADA A LOS ESTÁNDARES INTERNACIONALES DE OCDE
}

\section{ANÁLISE DO MARCO NORMATIVO DA ATUAÇÃO DENTRO DA REPÚBLICA ARGENTINA DAS SOCIEDADES OFF SHORE: ESPECIAL REFERENCIA OS PADRÕES INTERNACIONAIS DA OCDE}

Candela Noelia Villegas

Resumen: En este trabajo planteamos un análisis del marco normativo de actuación de las sociedades off shore dentro de la República Argentina en las diferentes dimensiones del derecho internacional privado. Pretendemos destacar las particularidades inherentes de este tipo social y su dinámica dentro de los centros financieros off shore para indagar los efectos que surgen como consecuencia de su accionar. Nos motiva la problemática, ya que en la actualidad se torna imprescindible el estudio de las diferentes aristas del tema $y$ profundizar el conocimiento de las formas de actuación de las sociedades off shore para encontrar soluciones acordes a las necesidades actuales, como un nuevo desafío del derecho internacional privado posmoderno.

Realizamos especial referencia a las principales acciones internacionales que se están llevando a cabo por los organismos internacionales especializados, especificamente por la Organización para la Cooperación y el Desarrollo Económico (OCDE) para concluir reflexionando sobre la obligatoriedad de esta fuente trasnacional en nuestro ordenamiento jurídico.

Resumo: Neste trabalho realizamos uma análise do marco normativo da atuação dentro da República Argentina das sociedades off shore nas diferentes dimensões do direito internacional privado. Pretendemos destacar as particularidades inerentes deste tipo social y sua dinâmica dentro dos centros financeiros off shore para indagar os efeitos que surgem como consequência de sua atuação. Nos motiva a problemática, já que na atualidade se faz necessário o estudo das diferentes arestas do tema

* Universidad Nacional del Córdoba, Argentina.

E-mail: candela_villegas@hotmail.com

Recibido: 09/01/2018. Aceptado: 05/02/2018. 
e aprofundar o conhecimento das formas de atuação das sociedades off shore para encontrar soluções acordes às necessidades atuais, como um novo desafio do direito internacional privado pós-moderno.

Realizamos uma especial referência às principais ações internacionais que se estão sendo levadas adiante pelos organismos internacionais especializados, em especial pela Organização para a Cooperação e Desenvolvimento Econômico (OCDE) para concluir refletindo sobre a obrigatoriedade desta fonte transacional em nosso ordenamento jurídico.

Palabras clave: Sociedades off shore, IGJ, OCDE, Fuente trasnacional, Soft law

Palavras-chave: Sociedades off shore, IGJ, OCDE, Fonte transacional, soft law

\section{INTRODUCCIÓN}

El auge de las sociedades off shore responde a una realidad social y económica directamente vinculada con los cambios en el tráfico comercial internacional y la facilitación de las comunicaciones. En un contexto de globalización económica como el actual, donde las principales características son la libre circulación de bienes y servicios, existe una tendencia natural a moverse traspasando los limites de las jurisdicciones territoriales con los fines de maximizar la obtención de beneficios empresariales. De esta manera, la internacionalización ${ }^{1}$ del capital genera una dispersión tal que resulta muy dificultoso su control y seguimiento.

El objetivo de este trabajo es analizar el marco normativo de actuación dentro de la República Argentina de las sociedades off shore en las diferentes dimensiones del derecho internacional privado, observar las características inherentes de este tipo social y su dinámica dentro de los centros financieros off shore. Así mismo, repasar las principales acciones internacionales que se están llevando a cabo por organismos especializados, específicamente por la Organización para la Cooperación y el Desarrollo Económico (OCDE) para concluir reflexionando sobre la obligatoriedad de esta fuente trasnacional en nuestro ordenamiento jurídico.

De esta manera, pretendemos brindar un panorama de la dinámica del funcionamiento de las sociedades off shore a partir de las distintas modalidades en que desarrollan su actividad. Sabemos que

1 Sobre el tema, nos explica Boaventura de Sousa Santos que "la globalización está teniendo un impacto uniforme en todas las regiones del mundo y en todos los sectores de actividades y que sus arquitectos, las empresas multinacionales, son infinitamente innovadores y tienen una capacidad organizativa suficiente para transformar la nueva economía global en una oportunidad sin precedentes". (DE SOUSA SANTOS, Boaventura. La caída del Angelus Novus: Ensayos para una nueva teoría social. Bogotá: Colección En Clave de Sur, 2003.p.193) 
se trata de una problemática cuyo estudio sistemático implicaría un análisis multidisciplinario ${ }^{2}$, abarcando estudios específicos en materia societaria, bancaria, tributaria, registral y de penal económico, pero nos enfocaremos en un estudio descriptivo de la temática desde la óptica del derecho internacional privado, con especial mención del diálogo entre sus fuentes.

Sobre la base de estos antecedentes, entendemos que se torna imprescindible el estudio de las diferentes aristas de la problemática y profundizar el conocimiento de sus formas de actuación para encontrar soluciones acordes a las necesidades actuales, representando la problemática uno de los nuevos desafíos que nos plantea el derecho internacional privado postmoderno. Como señala Sánchez Lorenzo ${ }^{3}$,

El derecho internacional privado presenta una ventaja metodológica, fruto de su carácter cosmopolita y dinámico, que nos permite adaptarnos a la realidad subyacente cambiante. Más que ningún otro saber jurídico, el derecho internacional privado ha evolucionado históricamente en busca de un paradigma con el que enfrentarse a su consustancial complejidad, al caos que introduce en la coherencia y la congruencia del cosmos jurídico el mero hecho de la pluralidad jurídica, de la existencia de distintos universos jurídicos.

\section{PUNTO DE PARTIDA: SITUACIÓN EN ARGENTINA}

La Ley General de Sociedades (LGS) $19.550^{4}$, en el artículo $118^{5}$ contempla la situación de las sociedades que constituidas en un Estado extranjero, pretenden realizar actividad en nuestro país, ya sea mediante

\footnotetext{
2 Profundizamos el estudio interdisciplinario de la problemática en el Congreso Internacional "Instituciones e interdisciplina. Alcances jurídicos, económicos y epistemológicos" y "III Workshop Nacional Metáfora y episteme: hacia una hermenéutica de las instituciones" (2017) Universidad Nacional de la Pampa. Trabajo titulado: Análisis de la actuación de las sociedades off Shore en la República Argentina, a la luz del derecho internacional privado y del derecho penal económico. Disponible en las actas del Congreso.

3 SÁNCHEZ LORENZO, Sixto Alfonso. "Estado democrático, Postmodernismo y derecho Internacional Privado". Revista de Estudios Jurídicos, 2010. No 10, p. 10.

4 Argentina. Ley No 19.550. Ley General de Sociedades. Texto ordenado por el Anexo del Decreto $N^{\circ} 841 / 84$ B.O. 30/03/1984. Denominación del Título sustituida por punto 2.1 del Anexo II de la Ley N ${ }^{\circ} 26.994$ B.O. 08/10/2014 Suplemento. Vigencia: $1^{\circ}$ de agosto de 2015, texto según art. $1^{\circ}$ de la Ley $N^{\circ} 27.077$ B.O. 19/12/2014.

5 Artículo 118. La sociedad constituida en el extranjero se rige en cuanto a su existencia y formas por las leyes del lugar de constitución.

Se halla habilitada para realizar en el país actos aislados y estar en juicio.

Para el ejercicio habitual de actos comprendidos en su objeto social, establecer sucursal asiento o cualquier otra especie de representación permanente, debe:

1) Acreditar la existencia de la sociedad con arreglo a las leyes de su país.

2) Fijar un domicilio en la República, cumpliendo con la publicación e inscripción exigidas por esta ley para las sociedades que se constituyan en la República;

3) Justificar la decisión de crear dicha representación y designar la persona a cuyo cargo ella estará.

Si se tratare de una sucursal se determinará además el capital que se le asigne cuando corresponda por leyes especiales.
} 
la forma de actos aislados, como de ejercicio habitual. Las exigencias según nos encuadremos en un supuesto legal o en el otro, varían significativamente, como así también los efectos que estas distintas formas de actuación generan.

El acto ejecutado por una sociedad constituida en el extranjero podrá ser calificado como acto aislado, o incluírselo en el concepto de ejercicio habitual, en virtud del criterio sentado por el artículo 118 de la LGS. Pero la cuestión no resulta tan simple como parece, ya que analizando las distintas posturas en doctrina y jurisprudencia nacional, encontramos que el límite entre ellos es muy frágil y la cuestión se torna muy delicada al momento de establecer los efectos que genera que el acto o los actos encuadren en un supuesto legal o en el otro ${ }^{6}$.

El sistema formulado por nuestro país no atiende a la naturaleza jurídica de la sociedad en sí, sino que se fundamenta en la naturaleza de los actos que la sociedad constituida en el extranjero realiza en nuestro país. La jurista Berta Kaller de Orchansky ${ }^{7}$ nos explica que se distinguen dos categorías de actos: a) de capacidad genérica, supuestos en cual impera la extraterritorialidad total ( para la realización de estos actos no se requiere que la sociedad se someta a la ley territorial de donde pretende actuar, bastará con justificar que se constituyó legalmente para que su capacidad sea reconocida de pleno derecho); b) de capacidad específica, actos referidos a la realización del objeto social y que tienden al cumplimiento de sus fines (prevalece aquí el sistema territorialista, por lo que para ejercer este tipo de actos deberá someterse a las leyes del país en que pretenda actuar). Podemos observar como de esta manera queda planteada la teoría intermedia que se refleja en el articulado de la LGS.

Determinadas sociedades amparándose en el artículo $1182^{\circ}$ párrafo no se inscriben en los registros correspondientes evitando la publicidad que correspondería por la entidad de actos que llevan a cabo, excediendo, a consideración de la Inspección General de Justicia, la calidad de acto aislado.

De las investigaciones de la Inspección General de Justicia ${ }^{8}$ (IGJ) surge que las sociedades off shore actualmente pueden ingresar a actuar

6 Para profundizar sobre la problemática de la calificación de los actos aislados vid: Villegas, Candela Noelia "Los Actos Aislados de las sociedades constituidas en el extranjero y la problemática de su calificación” Anuario XV (2013-2014) Centro de Investigaciones Jurídicas y Sociales. Buenos Aires: La Ley.2015.

7 KALLER DE ORCHANSKY, Berta. Nuevo Manual de Derecho Internacional Privado . Buenos Aires: Ed. Plus Ultra, 1995. p 426.

8 Inspección General de Justicia. La Inspección General de Justicia, organismo dependiente del Ministerio de Justicia y Derechos Humanos de la Nación, tiene la función de registrar y fiscalizar a las sociedades comerciales, sociedades extranjeras, asociaciones civiles y fundaciones que se constituyen en el ámbito de la Ciudad Autónoma de Buenos Aires. A su vez, es la encargada de la inscripción en el Registro Público de Comercio de las sociedades comerciales nacionales y extranjeras, las matrículas de los comerciantes, y de los auxiliares de comercio; ejercer el control federal de las sociedades de ahorro e implementar el Registro Nacional de Sociedades (Art. 3 Ley 22.315, Art. 2 Ley 26.047). 
en nuestro país mediante dos formas. La primera de ellas, es por la realización de actos aislados, que basta con la acreditación de su efectiva constitución en otro Estado y como consecuencia de ello se le reconoce de pleno derecho la personalidad jurídica. $\mathrm{O}$ a través de la instalación de sucursales, representaciones permanentes o participando de sociedades locales (supuesto permitido por el artículo $123^{9}$ ). Este último supuesto ha caído en desuso para las sociedades off shore, (al menos en gran medida en la Ciudad Autónoma de Buenos Aires) por las resoluciones dictada por IGJ.

Desde el año 2003 en adelante este organismo dictó Resoluciones Generales cuyo destinatario de aplicación son las sociedades constituidas en el extranjero. La Resolución General de la IGJ 7/2005 ${ }^{10}$ realiza una recopilación de la normativa vigente dictada por este organismo.

Para el primer supuesto de actuación al que hicimos referencia, el caso de los actos aislados, IGJ sancionó la Resolución 8/03, creando el denominado "Registro de Actos Aislados" con la finalidad de controlar y fiscalizar la actuación de los entes foráneos en nuestro país y evitar el abuso del acto aislado ${ }^{11} \mathrm{y}$ de esta manera le permitía al ente tomar conocimiento de los tipos de sociedades que ejercían estos actos en nuestro país. Sin embargo, esta cruzada que realizó la IGJ contra las sociedades off shore, si bien ha contribuido a disminuir el arribo de este tipo de sociedades, no resulta totalmente efectivo ya que el ámbito de legitimación de control de IGJ está limitado a la Ciudad Autónoma de Buenos Aires.

En el año 2015 IGJ realiza una nueva recopilación de todas las resoluciones vigentes a los fines de reordenar todo lo regulado por las resoluciones generales posteriores a la 7/2005 con miras a la armonización normativa y una necesaria actualización.

Conforme lo dispuesto por el artículo $218^{12}$ del Anexo "A" de la Resolución General I.G.J. No 07/2015, no se inscribirán en los términos de los artículos 118 o 123 de la Ley No 19.550, sociedades categorizadas como off shore o provenientes de jurisdicciones de ese carácter. Imponiéndoles la carga de adecuarse a la legislación argentina previamente a solicitar la inscripción conforme artículo 118 y 123 LGS.

9 Artículo 123. Para constituir sociedad en la República, deberán previamente acreditar ante el juez del Registro que se han constituido de acuerdo con las leyes de sus países respectivos e inscribir su contrato social, reformas y demás documentación habilitante, así como la relativa a sus representantes legales, en el registro Público de Comercio y en el registro Nacional de Sociedades por Acciones en su caso.

10 Argentina. Resolución General de la IGJ 7/2005. Título III "Sociedades Constituidas en el Extranjero", arts 188-250. B.O 25/08/05.

11 MARTÍNEZ, Silvina. "Sociedades extranjeras y el registro de actos aislados". La Ley 14/12/2005. LA LEY2006-A,1

12 Artículo 218. La Inspección General de Justicia no inscribirá a los fines contemplados en este Capítulo a sociedades "off shore" provenientes de jurisdicciones de ese carácter. Dichas sociedades, para desarrollar actividades destinadas al cumplimiento de su objeto y/o para constituir o tomar participación en otras sociedades, deben con carácter previo adecuarse íntegramente a la legislación argentina, cumpliendo al efecto con las disposiciones del título IV. 
En relación a los actos aislados, el artículo $262^{13}$ del Anexo "A" de la mencionada Resolución, impone a aquellas sociedades cuyos actos no revistan el "carácter de actos aislados" o "actos sometidos a investigación" deben adecuarse al régimen que establece la LGS para sociedades constituidas en la República, cumplimentando con todo lo exigido en el capítulo "Sociedad con domicilio o principal objeto destinado a cumplirse en la República. Adecuación a la ley argentina” de la Resolución 07/2015.

Este artículo deja sin mencionar un supuesto, el de las sociedades off shore que realizan actos aislados en nuestro país. El artículo 118 de la LGS en su $2^{\circ}$ párrafo habilita a la sociedad a "realizar en el país actos aislados y estar en juicio". Para llevarlos a cabo bastará con acreditar que se ha constituido conforme las leyes del lugar que le otorgó la personalidad jurídica y en Argentina nos limitamos a reconocerla y permitirle que realice los actos comprendidos en su capacidad genérica.

En este orden de ideas, planteado el marco normativo de la dimensión autónoma del derecho internacional privado argentino, y señalados los diferentes supuestos de actuación, continuaremos con el análisis de los centros financieros y las principales características de las sociedades off shore.

\section{3. ¿PARAÍSOS FISCALES O CENTROS FINANCIEROS?}

Para una mejor comprensión del tema y en función de las consideraciones anteriores, resulta imprescindible diferenciar algunos conceptos previamente a ingresar en la problemática específica de las sociedades off shore.

En una primera instancia deberíamos comenzar por echar luz sobre los conceptos "paraísos fiscales" y "centros financieros off shore". "Paraísos fiscales" es un concepto que se acuño a fines del siglo XIX, más específicamente en el comienzo del S.XX y tuvo vigencia hasta la década de los '90, que se empieza a hablar de centros financieros off shore. Los paraísos fiscales respondían principalmente a las necesidades de obtener regímenes de baja o nula tributación, secreto bancario y anonimato en las relaciones financieras. Supone un cierto carácter pasivo, cuyos fines son el ocultamiento y anonimato, en contraposición a los centros financieros off shore que detentan una actividad más activa frente al dinámico tráfico comercial internacional. Es a manera de evolución que surgen los centros financieros off shore, representando una versión modernizada de los paraísos fiscales.

Las distinciones que se realizan responden más a cuestiones

13 Artículo 262. Las sociedades "off shore", cuando no corresponda admitir el carácter de aislado del acto o actos sometidos a investigación conforme al presente Capítulo, serán intimadas únicamente a los fines de su adecuación a las disposiciones de la Ley No 19.550 aplicables a las sociedades constituidas en la República, debiendo cumplir al efecto con el procedimiento y requisitos establecidos en el Capítulo IV. 
teóricas y evolución histórica que a una diferencia desde el punto de vista práctico.

También se debe tener en cuenta que denominar a estas jurisdicciones de una manera $\mathrm{u}$ otra, genera un impacto diferente en la sociedad. Por una cuestión de imagen son peores vistos los paraísos fiscales que los centros financieros off shore.

Resulta complejo trazar una línea divisoria entre ellos, ya que se trata de términos que en doctrina no han sido conceptualizados con uniformidad. Por lo que entenderemos que los centros financieros off shore resultan una evolución de los paraísos fiscales y continuaremos refiriéndonos a ellos con la última denominación. Y para ello seguiremos el concepto acuñado por el Fondo Monetario Internacional que define a los centros financieros off shore como:

Centros en los que la mayor parte de las transacciones financieras de la balanza comercial se realizan con personas o compañías no residentes en el centro financiero off shore, donde las transacciones pueden iniciarse desde cualquier parte, y donde la mayoría de las instituciones implicadas son controladas por no residentes. Así, muchos de los centros financieros off shore tienen las siguientes características:

1. Jurisdicciones que tienen instituciones financieras dedicadas principalmente a negocios con no residentes;

2. Sistemas financieros con activos y obligaciones externas desproporcionados con la intermediación financiera de la economía doméstica.

3. Más popularmente, los centros que ofrecen algunas o todas las siguientes oportunidades: impuestos bajos o cero; Regulación financiera moderada o ligera; El secreto bancario y el anonimato ${ }^{14}$. (Traducción de la autora)

Es importante también distinguir los centros financieros off shore de los puramente "centros financieros". Estos últimos, no llegan a tener todas las características inherentes de un centro financiero off shore, si bien poseen algunas de ellas, no resultan suficientes para incluirlos en esa definición.

Las características esenciales de cada uno de ellos son ampliamente conocidas por las empresas que realizan su planificación fiscal en función de los beneficios que desean obtener y así escogen el centro donde pretenden actuar.

Podemos dividir en tres grandes grupos a los centros financieros off shore $^{15}$ : el primero al que haremos referencia es aquellas jurisdicciones

14 INTERNATIONAL MONETARY FUND (IMF). “Offshore Financial Centers The Role of the IMF”. Junio, 2000.

15 Clasificación según: WARTHON, UNIVERSITY OF PENNSYLVANIA. "Paraísos fiscales y empresas off shore: La fiscalidad internacional al servicio de la empresa". 2015. Disponible en: $<$ https://www.knowledgeatwharton.com.es/article/paraisos-fiscales-y-empresas-off shore-lafiscalidad-internacional-al-servicio-de-la-empresa/> (Recuperado: 8 de junio de 2017). 
que se caracterizan por no gravar con impuestos a las ganancias, ni a las sociedades, ni a las sucesiones; constituyen ejemplo de ellos las Islas Caimán, Bahamas, Dubai, etc. Un segundo grupo tiene como rasgo distintivo que eximen de tributar por las rentas generadas en el extranjero, sólo gravan los beneficios obtenidos locamente, siempre que la fuente de ingresos extranjeros no tenga origen en una actividad empresarial local. Este es el caso que nuclea a países como Panamá, Singapur, Costa Rica, entre otros. Y finalmente encontramos centros que procuran beneficios impositivos para situaciones determinadas, estrictamente definidas o que se valen de la utilización de tratados de doble imposición ${ }^{16}$. Las situaciones especiales pueden ser por ejemplo las ganancias de capital, royalties, etc. Los países que encontramos con este régimen: Reino Unido, Holanda, Dinamarca, Malta, etc ${ }^{17}$.

\section{SOCIEDADES OFF SHORE: PRINCIPALES CARACTERÍSTICAS}

Bajo la premisa de que la realidad actual presenta a los empresarios la necesidad de ser eficientes en un mundo competitivo y que las grandes crisis económicas y el modelo de economía global exigen por parte de los sujetos comerciales, no sólo eficiencia en su producción, sino también optimización de todos los recursos disponibles, es que los centros financieros off shore emergen y alcanzan el grado de importancia que tienen en la actualidad.

Cada centro financiero off shore detenta notas tipificantes propias y ventajas particulares. Estas características son tenidas en cuenta por los inversionistas al momento de realizar la elección de la jurisdicción y del tipo societario del que se valdrán en función de los beneficios que desean obtener para sus intereses.

De este modo la planificación fiscal ingresa como tema principal en la agenda empresarial. En la búsqueda de regímenes fiscales más favorables donde deslocalizar sus inversiones, aspiran a instalarse en jurisdicciones que habilitan la constitución de sociedades off shore.

Sumado a esto, resultan también atractivos, los beneficios del

16 "Inicialmente, los Convenios de doble imposición tenían como misión delimitar o condicionar el ejercicio de las potestades tributarias tal como venían reconocidas en la legislación tributaria interna de dichos Estados, pero de forma cada vez más importante determinadas cláusulas tributarias internas son diseñadas a modo de complemento, reacción o prevención frente al uso inadecuado o indeseado de los Convenios de doble imposición por parte de determinados contribuyentes. A su vez, y aunque no existan estudios fiables al respecto, modulan el comportamiento de las empresas que abordan el fenómeno de la globalización económica ajustando sus estructuras organizativas, productivas y de decisión al entramado que forma dicha red con vistas a minimizar los costes tributarios globales y, así, maximizar los resultados económicos" GARCIA PRATS, Francisco Alfredo. "Los modelos de convenios, sus principios rectores y su influencia sobre los convenios de doble imposición". Crónica Tributaria, España, N 133/2009, 2009. P. 102.

17 WARTHON, UNIVERSITY OF PENNSYLVANIA. "Paraísos fiscales y empresas off shore: La fiscalidad internacional al servicio de la empresa". 2015. 
anonimato, el secreto en las transacciones, la fácil y ágil constitución, los mínimos requisitos a acreditar, entre otras ventajas que ofrece la utilización de esta clase de sociedades.

La denominación sociedades off shore tiene su origen en el idioma inglés. El término off shore se utiliza para designar la línea divisoria entre el agua del mar y la tierra. Se trata de un término que no aparece en los diccionarios, pero que la costumbre ha instaurado en ámbito de mercado comercial.

Las sociedades off shore, en primera instancia son entes ideales. $\mathrm{Su}$ finalidad es actuar solamente fuera de las fronteras del lugar de su constitución. Tienen vedado o sumamente limitado el desarrollo de su objeto social en el país que les otorga el reconocimiento de la personalidad jurídica. Están destinadas a una actuación exclusivamente extraterritorial. El Dr. Vítolo se refiere a éstas sociedades diciendo que

constituyen una perversa manera de crear sujetos dentro de una jurisdicción para remitirlos como "enviados" a ejercer su actividad "fuera de casa" bajo la promesa de que podrán obtener en el país de destino un reconocimiento para poder realizar allí todo aquello que no le es permitido realizar en su propio país de origen, sin embargo ese país de origen que no le permite actuar dentro de su territorio, protegerá a los socios de la sociedad off shore con el secreto y el anonimato frente a las preguntas de cualquier tercero o Estado del mundo que desee hacer sobre ellos ${ }^{18}$.

Este tipo social permite a los no residentes servirse de los beneficios que ofrecen los paraísos fiscales, donde encuentran normativas destinadas a proteger el anonimato de los socios, principalmente porque facilitan la entrega de acciones al portador, sin realizar ningún tipo de registración en los libros societarios ni en registros del Estado, situación que impide el conocimiento de las verdaderas personas que resultaron propietarias de esos títulos.

En relación al capital social las sumas que se exigen son extremadamente bajas, en consecuencia, para la constitución de una de estas sociedades no es necesario el aporte de un monto significativo, por lo general existen tasas fijas que deben abonar los socios.

Otra característica atractiva de estos centros financieros es que facilitan la designación de personas interpósitas (por lo general insolventes) en los negocios propios, ya que para el nombramiento de quienes estarán a cargo de los órganos de administración las exigencias legales son prácticamente nulas.

Todas estas flexibilidades permiten que acontezcan situaciones como las que hemos observado en los últimos años, donde empresarios

18 VÍTOLO, Daniel Roque. La personalidad jurídica de las sociedades comerciales. Su limitación en los casos de utilización indebida y fraude. Buenos Aires: Ed. Errepar, 2010. p. 278. 
que pretenden actuar en Argentina esquivan las responsabilidades personales que se le podría atribuir, a través de la constitución, con fines deshonestos, de sociedades off shore en jurisdicciones más flexibles ${ }^{19}$, pero exclusivamente destinadas a actuar en otro Estado.

Debemos tener en cuenta que si las personas humanas o jurídicas quieren realizar una planificación fiscal favorable a su economía, en la cual se disminuya la carga tributaria, existen mecanismos legales a nivel internacional para lograrlo, no siendo siempre éstos ilícitos. El gran problema de la utilización de las sociedades off shore es la intención de valerse de esta figura para evadir el régimen legal que le corresponde aplicar.

Para disuadir la utilización de sociedades off shore, existen numerosas medidas preventivas, tanto a nivel interno como a nivel internacional, generadas por la actividad de los organismos internacionales especializados en la problemática.

Desde la óptica internacional la creación de leyes modelos, donde se plasman los principales criterios que surgen de los estándares internacionales, sirven como fuente de inspiración para que esos parámetros sean incorporados a través de la legislación interna a nuestro sistema jurídico y en consecuencia tornarlos obligatorios. También funcionan como medidas preventivas las Recomendaciones que realizan las diferentes organizaciones internacionales (principalmente la Organización para la Cooperación y el Desarrollo Económicos y el Grupo de Acción Financiera) sobre la temática, que si bien no son vinculantes para los Estados, sirven a los fines de ejercer presión en la sanción de leyes internas acordes a las recomendaciones.

\section{LAS ACCIONES INTERNACIONALES DE OCDE}

La Organización para la Cooperación y el Desarrollo Económicos $(\mathrm{OCDE})^{20}$ tiene como misión promover políticas que mejoren el bienestar económico y social de las personas alrededor del mundo.

En relación a los instrumentos legales de los cuales se vale

19 Con la finalidad de proporcionar un abanico de posibilidades más flexible y atraer de esta manera inversiones extranjeras que se traduzcan en una mayor competitividad internacional, diferentes jurisdicciones, que se caracterizan por una fuerte carga tributaria han ido adoptando figuras legales que resulten atractivas a los grandes empresarios. Existen numerosos ejemplos que pueden enunciarse, a modo ilustrativo señalaremos el caso de las ETVE españolas: Artículos 116 de la Ley del Impuesto sobre Sociedades en relación a las Entidades de Tenencia de Valores Extranjeros: "Aquellas cuyo objeto social comprenda la actividad de gestión y administración de valores representativos de los fondos propios de entidades no residentes en territorio español, mediante la correspondiente organización de medios materiales y personales".

20 Fundada en 1961, la Organización para la Cooperación y el Desarrollo Económicos (OCDE) agrupa a 34 países miembros: Alemania, Australia, Austria, Bélgica, Canadá, Chile, Corea, Dinamarca, España, Estados Unidos, Eslovenia, Estonia, Finlandia, Francia, Grecia, Hungría, Irlanda, Islandia, Israel, Italia, Japón, Letonia, Luxemburgo, México, Noruega, Nueva Zelanda, Países Bajos, Polonia, Portugal, Reino Unido, República Checa, Suecia, Suiza, Turquía. 
la Organización, podemos distinguir dos tipos, las decisiones y recomendaciones. Las decisiones se caracterizan por no constituir tratados internacionales, sin embargo, para los miembros que no se abstengan expresamente son legalmente obligatorias. Su implementación interna se realiza conforme los regímenes constitucionales propios. Las recomendaciones, por su parte, aunque carecen de fuerza vinculante, se espera que los miembros las incorporen a sus ordenamientos internos por su fuerte legitimación técnica ${ }^{21}$.

Debemos destacar que la Organización está a la vanguardia de los esfuerzos emprendidos para ayudar a los gobiernos a entender y responder a los cambios y preocupaciones del mundo actual, como el gobierno corporativo y la economía de la información ${ }^{22}$. Conduce una gran lucha en la erradicación de los paraísos fiscales y en la eliminación de las sociedades off shore. Como consecuencia de ello, la OCDE ofrece un Foro donde los gobiernos puedan trabajar conjuntamente para compartir experiencias y buscar soluciones a los problemas comunes ${ }^{23}$.

La iniciativa de combatir a los paraísos fiscales y a la utilización de sociedades off shore con fines de defraudación, no le ha resultado fácil. Los Estados en los cuales se ubican los centros financieros que facilitan estas operaciones, en su mayoría, dependen económicamente de la constitución de este tipo societario e intentan ser focos cada vez más atractivos hacia las inversiones, ofreciendo tentadoras ventajas.

En consecuencia, se tornó imprescindible para la OCDE repensar nuevas estrategias frente a este fenómeno, deduciendo que resultaría más efectivo si lograban la colaboración por parte de estos Estados en la facilitación de la información sobre las diferentes actividades y operaciones financieras que se realicen bajo su jurisdicción. Para ello, se reactiva el Foro de Transparencia Global, el cual confeccionó la lista de países colaboradores ${ }^{24}$, que son aquellos que cooperan brindando información y colaborando en los trabajos relativos a la transparencia y el intercambio de información con fines fiscales.

Una de las principales líneas de trabajo de OCDE, ha sido el desarrollo de estándares internacionales, que están siendo aprobados casi universalmente, e implementándose en los países miembros. A los fines de lograr una implementación eficaz, la OCDE se enfoca en las estrategias para confeccionar mecanismos de revisión.

21 LOAIZA KEEL, Carlos. "Uruguay miembro de la OCDE: Un camino al desarrollo". Pharos Academia Nacional de Economía. Universidad de Montevideo. Montevideo:Ed. Mastergraf. 2016. 22 OCDE. "Foro Global sobre Transparencia e Intercambio de Información con Fines Tributarios. Informe de la Revisión de Pares: Argentina 2012: Combinado: Fase $1+$ Fase 2 (Spanish version)", OECD Publishing.

23 Información disponible en: “Acerca de la Organización para la Cooperación y el Desarrollo Económicos (OCDE)": Disponible en: <http://www.oecd.org/centrodemexico/laocde/> (Consultado: 25 de Julio de 2017.

24 En el Informe de progreso del 2 de Abril de 2012 figuran la lista de de jurisdicciones cooperativas. 
Los estándares establecen el intercambio internacional de información que sea previsiblemente relevante para la administración o aplicación de la legislación tributaria interna de los Estados miembros. Si bien con ello no se habilita a solicitar información de manera masiva (las conocidas fishing expeditions), los Estados deben facilitar toda aquella información que sea previsiblemente relevante.

Con el objetivo principal de establecer un mecanismo confiable de revisión que tenga como finalidad, facilitar la implementación de los estándares internacionales y de cooperar en la tarea de adaptar los criterios sugeridos sobre transparencia e intercambio de información, los miembros del Foro Global se someten a un sistema de evaluación. Este proceso se realiza en dos fases. En la denominada "Fase 1" se analiza el marco normativo relativo al intercambio de información del que goza una determinada jurisdicción. En la "Fase 2" se evalúan las implicancias prácticas de la aplicación de ese marco normativo, es decir su efectiva aplicación.

Es así como el Foro Global vigila el cumplimiento de los estándares internacionales a través de este proceso de revisión por pares, siempre con miras a respetar la igualdad de condiciones entre todos los Estados. El Foro Global se constituye como el principal organismo internacional encargado de velar por la aplicación de las normas internacionalmente acordadas.

Es importante destacar que últimamente, como consecuencia de la presión que se efectúa desde el G20, cada vez son más los países que adoptan medidas de colaboración en materia de transparencia fiscal y lucha contra la evasión, ya sea revisando los tratados que tienen ratificados como también celebrando y negociando nuevos acuerdos.

En la reunión realizada en Tiflis ${ }^{25}$, en Noviembre de 2016, se indicó en la Declaración de Resultados que el Foro Global se reafirma

en su papel de principal organismo internacional en cuanto a transparencia e intercambio de información con fines fiscales. Con el objeto de no socavar los

25 Participaron de la reunión: Alemania; Antigua y Barbuda; Arabia Saudita; Argentina; Austria; Bahamas; Barbados; Baréin; Bélgica; Belice; Bermudas; Brasil; Bulgaria; Camerún; Canadá; Catar; Chile; Colombia; Corea; Costa Rica; Dinamarca; Emiratos Árabes Unidos; España; Estados Unidos; Estonia; Finlandia; Francia; Georgia; Ghana; Gibraltar; Guernesey; Hong Kong (RAE China); Hungría; India; Indonesia; Irlanda; Isla de Man; Islas Caimán; Islas Cook; Islas Marshall; Islas Turcas y Caicos; Islas Vírgenes Británicas; Italia; Japón; Jersey; Kazajistán; Kenia; Kuwait; Letonia; Liechtenstein; Lituania; Luxemburgo; Macao (RAE China); Malasia; Malta; México; Moldavia; Mónaco; Noruega; Países Bajos; Pakistán; Panamá; Paraguay; Perú; Polonia; Portugal; Reino Unido; República Dominicana; República Eslovaca; República Popular China; Rumanía; Rusia; Samoa; San Cristóbal y Nieves; San Marino; Senegal; Seychelles; Singapur; Sudáfrica; Suecia; Suiza; Trinidad y Tobago; Turquía; Uganda; Uruguay. Banco Europeo de Inversiones (BEI); Banco Europeo de Reconstrucción y Desarrollo (BERD); Centro de Encuentros y Estudios de Directores de las Administraciones Tributarias (CREDAF, por sus siglas en francés); Centro Interamericano de Administraciones Tributarias (CIAT); Comisión Europea (CE); Corporación Financiera Internacional (CFI); Foro Africano de Administración Tributaria (ATAF, por sus siglas en inglés); Grupo de Acción Financiera (GAFI); Grupo del Banco Mundial (GBM); Naciones Unidas (ONU); Organización de Cooperación y Desarrollo Económicos (OCDE); Secretaría de la Commonwealth. 
esfuerzos realizados por las jurisdicciones para cumplir los estándares, se hizo un llamamiento para que todas las iniciativas relacionadas con la transparencia fiscal, tales como la elaboración de listas de jurisdicciones no cooperativas, se ajusten plenamente al trabajo del Foro Global, incluidos los resultados del proceso de evaluación abreviado" 26 .

En esta instancia, es necesario detenernos sobre un punto importante de análisis, la obligatoriedad y fuerza vinculante de los parámetros que se fijan a nivel internacional por organismos especializados (OCDE como uno de los principales referentes en la lucha contra las sociedades off shore), y repensar ¿Las recomendaciones de OCDE, constituyen fuente de derecho internacional privado? ¿Representan parámetros vinculantes para los Estados miembros y no miembros de las organizaciones? ¿Cuál es su impacto en el derecho interno argentino?

Podríamos comenzar señalando que en principio, las recomendaciones de OCDE implicarían fuente de derecho internacional privado de dimensión trasnacional. Siguiendo el criterio de Dreyzin de Klor "esta fuente se caracteriza por la carencia de coercibilidad al no existir sanción por un poder público con potestad legislativa" ${ }^{27}$. Nos explica la jusrista en relación a los usos típicos del ámbito trasnacional que éstos

sobreviven de forma autónoma a modo de soft law o normas narrativas...esta fuente se materializa en recomendaciones, dictámenes, códigos de conducta y principios, entre otros instrumentos que no tienen poder de vinculación directa, aunque influyen tanto en el desarrollo legislativo futuro, como así también en calidad de referentes específicos en la actuación judicial ${ }^{28}$.

Esto implica que el poder legislativo de cada Estado podría incorporar a su normativa nacional los estándares fijados internacionalmente. El soft law, se manifiesta en la proliferación de leyes modelo elaboradas en foros internacionales, las que pueden ser tenidas en cuenta por el legislador nacional y por el intérprete ${ }^{29}$.

Frente a este panorama mundial, se han ido adaptando algunas de las jurisdicciones (antes calificadas de paraísos fiscales), reconvirtiéndose, e incorporando en sus ordenamientos internos, normativas favorables a mecanismos de control frente al fraude organizado y mostrando

26 "9th Meeting of the Global Forum on Transparency and Exchange of Information for Tax Purposes" (Foro Global sobre la Transparencia y el Intercambio de Información con Fines Fiscales). Declaración de Resultados. 2 y 4 de Noviembre de 2016, Tblisi, Georgia. Disponible en: <https://www.oecd.org/tax/transparency/about-the-global-forum/meetings/declaracionresultados-reunion-foro-global-2016.pdf >. Consultado: 12 de Junio de 2017.

27 DREYZIN DE KLOR, Adriana. El derecho Internacional Privado Actual. Tomo I. Buenos Aires: Ed. Zavalía, 2015, p. 63

28 Ibíd., p. 64.

29 Ídem. 
predisposición a los fines de adaptarse a los mecanismos de cooperación internacional en materia de intercambio de información.

\section{CONCLUSIÓN}

Desincentivar la utilización de las sociedades off shore con fines de fraude requiere de la unión de esfuerzos internacionales y de la consecuente toma de medidas internas en cada país.

Paulatinamente, los países identificados como centros financieros off shore, se han ido adaptando a los criterios que se fijan a nivel internacional y avanzando en cuestiones de cooperación internacional en intercambio de información, especialmente cuando ocurren situaciones en las que se encuentran vinculados delitos de blanqueo de capitales, narcotráfico y trata de personas. Sin embargo, queda un largo camino por recorrer.

La reticencia de las jurisdicciones que protegen con el anonimato y el secreto, incluso frente a situaciones como las anteriormente enunciadas, conlleva que las solicitudes de información y cooperación jurisdiccional entre países deban fundarse debidamente, de lo contrario son rechazadas de manera inmediata por el país requerido.

En los días que corren sabemos que hablar de las sociedades off shore implica una connotación negativa para la sociedad, como consecuencia de los numerosos escándalos que han ocurrido a nivel mundial y que se han dado a conocer mega casos de corrupción que envolvieron a figuras políticas y empresariales de todo el mundo.

Como contrapartida al uso fraudulento de las sociedades off Shore resulta importante aclarar que la constitución de una sociedad de este tipo es legal si se realiza conforme las normativas del lugar de constitución. El núcleo problemático de esta cuestión gira en torno a la utilización de esta figura como un medio para evadir la aplicación del orden jurídico que hubiese correspondido, "cuando en un intento de marginación de la legislación interna, se practica o se simula practicar desde el exterior una operatoria, se generan siempre sobre la actividad económica del país efectos nocivos" ${ }^{\text {"30 }}$.

En el caso de Argentina, es destacable la actuación de la IGJ, quien protagoniza una gran tarea en la erradicación de las maniobras fraudulentas que se realizan a través de estas sociedades.

Para desbaratar el fraude organizado es preciso que la tarea quede a cargo de operadores jurídicos especializados, con la finalidad de lograr una adecuada coordinación y respeto de las pautas que orientan la política exterior argentina en materia de obligaciones internacionales. Adoptar los criterios fijados por los organismos internacionales de los cuales somos miembros, resulta fundamental, no sólo por la responsabilidad

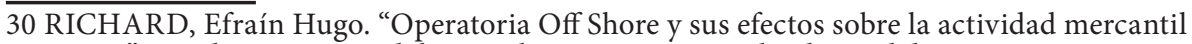
argentina”. Academia Nacional de Derecho y Ciencias Sociales de Córdoba. 
internacional que nos compete, sino también porque se fijan pautas que aúnan esfuerzos mundiales en búsqueda de soluciones a un problema actual que genera grandes perjuicios.

Para que un Estado pueda trazar un camino de crecimiento y una adecuada inserción internacional es imprescindible que cuente en su ordenamiento jurídico con normas claras y coherentes, sistemáticamente armonizadas. Es fundamental un régimen jurídico preciso, que recepte los estándares internacionales y que nos muestre al mundo como un Estado confiable y dotado de seguridad jurídica.

Es importante que tanto el legislador nacional, como los jueces, entendidos como últimos intérpretes sean receptivos de las acciones internacionales. Estamos frente a escenarios de cambios, las necesidades actuales nos obligan a receptar las acciones internacionales que se están llevando a cabo en esta materia para poder hablar de competitividad internacional entre países.

\section{AGRADECIMIENTOS}

Agradezco profundamente a mi directora de tesis doctoral, Prof. Dra. Adriana Dreyzin de Klor por su valiosa guía, comentarios y sugerencias en este trabajo.

\section{REFERENCIAS BIBLIOGRÁFICAS}

DE SOUSA SANTOS, Boaventura. La caída del Angelus Novus: Ensayos para una nueva teoría social. Bogotá: Colección En Clave de Sur, 2003.

DREYZIN DE KLOR, Adriana. El derecho Internacional Privado Actual. Tomo I. Buenos Aires: Ed. Zavalía, 2015.

FERNÁNDEZ ROZAS, José Carlos y SÁNCHEZ LORENZO, Sixto Alfonso. Derecho Internacional Privado. 9a ed. Madrid: Ed. Civitas, 2016.

GLOBAL FORUM ON TRANSPARENCY AND EXCHANGE OF INFORMATION FOR TAX PURPOSES. 9th Meeting. Declaración de Resultados. 2 - 4 de Noviembre de 2016, Tblisi, Georgia. Disponible en: <http://www.oecd.org/centrodemexico/ foroglobaldelaocdesobretransparenciaeintercambiodeinformacion.htm>

GARCIA PRATS, F.A. (2009) Francisco Alfredo. "Los modelos de convenios, sus principios rectores y su influencia sobre los convenios de doble imposición”. Crónica Tributaria, España, $N^{\circ}$ 133/2009, 2009. Disponible en: <http://www.ief.es/documentos/recursos/publicaciones/ fiscalidad_internacional/133_Garcia.pdf $>$

INTERNATIONAL MONETARY FUND (IMF). “Offshore Financial 
Centers The Role of the IMF". Junio, 2000. Disponible en: <http://www. imf.org/external/ns/search.aspx?NewQuery=off+shore\&submit=>

KALLER DE ORCHANSKY, Berta. Nuevo Manual de Derecho Internacional Privado. Buenos Aires: Ed. Plus Ultra, 1995.

LOAIZA KEEL, Carlos. "Uruguay miembro de la OCDE: Un camino al desarrollo". Pharos Academia Nacional de Economía. Universidad de Montevideo Montevideo:Ed. Mastergraf, 2016.

MARTÍNEZ, Silvina. "Sociedades extranjeras y el registro de actos aislados”. La Ley 14/12/2005. LA LEY2006-A, 1.

OCDE. "Foro Global sobre Transparencia e Intercambio de Información con Fines Tributarios. Informe de la Revisión de Pares: Argentina 2012: Combinado: Fase 1 + Fase 2 (Spanish version)", OECD Publishing. Disponible en: <http://dx.doi.org/10.1787/9789264202122-es>

OCDE, "Acerca de la Organización para la Cooperación y el Desarrollo Económico" Disponible en: <http://www.oecd.org/centrodemexico/laocde/>

ROZO, Carlos y AZAMAR, Aleida. "El G20 en Los Cabos. Oportunidad perdida para el cambio necesario. Problemas de Desarrollo". Problemas del Desarrollo. Science Direct, EBSCOhost. México, 2003, vol 44, n 175.

RICHARD, Efraín Hugo. "Operatoria Off Shore y sus efectos sobre la actividad mercantil argentina”. Academia Nacional de Derecho $y$ Ciencias Sociales de Córdoba. Disponible en: <http://www.acaderc.org. ar/doctrina>

SÁNCHEZ LORENZO, Sixto Alfonso. "Estado democrático, Postmdernismo y derecho Internacional Privado". Revista de Estudios Jurídicos, 2010, no 10.

VÍTOLO, Daniel Roque. La personalidad jurídica de las sociedades comerciales. Su limitación en los casos de utilización indebida y fraude. Buenos Aires: Ed. Errepar, 2010.

WARTHON, UNIVERSITY OF PENNSYLVANIA. "Paraísos fiscales y empresas off shore: La fiscalidad internacional al servicio de la empresa". 2015. Disponible en: <https://www.knowledgeatwharton.com.es/article/ paraisos-fiscales-y-empresas-off shore-la-fiscalidad-internacional-alservicio-de-la-empresa/> 


\section{RESUMEN BIOGRÁFICO}

Candela Noelia Villegas es Abogada. Escribana. Doctoranda en Derecho y Ciencias Sociales (Facultad de Derecho, UNC). Becaria doctoral de CONICET en el Centro de Investigaciones Jurídicas y Sociales CIJSCONICET UNC. Maestranda en Derecho y Argumentación (Facultad de Derecho, UNC). Adscripta en las Cátedras de Derecho Internacional Privado y de Derecho Privado IV (Sociedades Comerciales) Facultad de Derecho, UNC. Asistente de Investigación Secyt (UNC). 